\title{
Listeria monocytogenes en manipuladores de alimentos: un nuevo enfoque para tener en cuenta en los peligros de la industria alimentaria
}

\author{
Ángela Bibiana Muñoz, José Antonio Chaves, Edna Catering Rodríguez, María Elena Realpe \\ Grupo de Microbiología, Subdirección Red Nacional de Laboratorios, Instituto Nacional de Salud, \\ Bogotá, D.C., Colombia
}

\begin{abstract}
Introducción. Las enfermedades transmitidas por alimentos constituyen un problema sanitario de alto impacto. Listeria monocytogenes se asocia frecuentemente con contaminación de alimentos lácteos y cárnicos. Vigilar su presencia en varios puntos de esta producción, es vital para controlar la diseminación del microorganismo.

Objetivo. Determinar la prevalencia de L. monocytogenes en manipuladores de alimentos de lácteos y cárnicos en 10 departamentos de Colombia y buscar la asociación entre la presencia del microorganismo y posibles factores de riesgo.

Materiales y métodos. Se llevó a cabo un estudio descriptivo de corte transversal, en el cual se determinó la presencia de L. monocytogenes en muestras de materia fecal y frotis de manos, provenientes de 1.322 manipuladores de alimentos. Se aplicó un cuestionario para conocer posibles factores de riesgo, y se llevó a cabo un análisis estadístico para determinar frecuencias y establecer relaciones entre los posibles factores de riesgo y el estado del portador.

Resultados. Hubo $138(10,4 \%)$ manipuladores de alimentos que resultaron positivos para $L$. monocytogenes y se encontró asociación estadísticamente significativa entre la presencia del microorganismo y "no conocer el concepto de contaminación cruzada" $\left(\mathrm{OR}\left(\mathrm{IC}_{95 \%}\right)=1,518 ; p=0,004\right)$, así como con "no practicar procedimientos de limpieza y desinfección adecuados" (OR $\left(\mathrm{IC}_{95 \%}\right)=1,292$; $\mathrm{p}=0,005)$.

Conclusión. Se estableció una prevalencia de portadores de L. monocytogenes entre manipuladores de alimentos de derivados lácteos y cárnicos, y se logró asociar las prácticas higiénicas de los manipuladores de alimentos como factores de riesgo. El estudio se convierte en una herramienta útil para la vigilancia, la caracterización epidemiológica y el planteamiento de estrategias de control de este microorganismo.
\end{abstract}

Palabras clave: Listeria monocytogenes, enfermedades transmitidas por alimentos, productos lácteos, productos de la carne, manipulación de alimentos.

doi: http://dx.doi.org/10.7705/biomedica.v33i2.716

Listeria monocytogenes in food handlers: a new approach to address the dangers in food industry

Introduction: Foodborne diseases are a high-impact health problem. Listeria monocytogenes is frequently associated with contaminated meat and dairy foods. Monitoring their presence at various stages during production is important to control the spread of this microorganism. Objective: To determine the prevalence of $L$. monocytogenes in meat and dairy food handlers in ten departments of Colombia and to find an association between the presence of this microorganism and possible risk factors.

Materials and methods: A cross sectional study was carried out to determine the presence of $L$. monocytogenes in fecal samples and swabs taken from the hands of 1322 food handlers. A questionnaire was applied to determine possible risk factors and a statistical analysis was carried out to determine frequencies and relationships between potential risk factors and the presence of $L$. monocytogenes. Results: $138(10.4 \%)$ food handlers were positive for L. monocytogenes and a statistically significant association was found between the presence of this microorganism and the following risk factors: "

\section{Contribución de los autores:}

Ángela Bibiana Muñoz y José Antonio Chaves escribieron el proyecto para financiación y procesaron las muestras clínicas. Ángela Bibiana Muñoz hizo el análisis estadístico.

Edna Catering Rodríguez hizo el montaje e interpretación de pruebas de sensibilidad antibiótica.

María Elena Realpe revisó el proyecto.

Todos los autores participaron en la toma de muestras clínicas y en la revisión del documento. 
Lack of knowledge about the concept of cross-contamination" (OR ( CI 95\%) $=1.518 \mathrm{P}=0.004)$, and "Not practicing cleaning and disinfection procedures appropriately" (OR ( Cl 95\%) $=1.292 \mathrm{P}=0.005)$ Conclusion: Prevalence of $L$. monocytogenes carriers was established among meat and dairy food handlers and food handlers practices were associated as risk factors for carrier condition. This study is a useful tool for the monitoring, epidemiological characterization and definition of strategies to control this pathogen.

Keywords: Listeria monocytogenes, foodborne diseases, dairy products, meat products, food doi: http://dx.doi.org/10.7705/biomedica.v33i2.716 handling.

Las enfermedades transmitidas por alimentos constituyen uno de los problemas sanitarios más comunes y de mayor impacto sobre la salud de las personas en el mundo. Afectan principalmente a la población vulnerable y trae consigo perjuicios, no solo para la salud humana, sino también para el comercio, el turismo y el sector agroindustrial (1).

Según los datos de la Organización de las Naciones Unidas para la Alimentación y la Agricultura (FAO) y la Organización Mundial de la Salud (OMS), se estima que la región latinoamericana experimentó, al menos, 6.000 brotes de diversos tipos de enfermedades de origen alimentario entre 1993 y 2002, los cuales, junto con un número mayor de casos aislados de enfermedades provocadas por los alimentos o agua, causaron en la región unas 57.000 muertes en 2004. Sin embargo, según los expertos, esta estimación se encuentra todavía muy por debajo de la incidencia real del problema (2).

Los reportes sobre enfermedades transmitidas por alimentos en Colombia permiten evidenciar que, entre los alimentos procesados, los productos cárnicos y los productos lácteos son los de mayor consumo en el país y los que frecuentemente están relacionados como fuente de contaminación con microorganismos patógenos como Listeria monocytogenes (3). Este microorganismo es una bacteria ubicua que ha sido conocida como patógeno para animales desde comienzos del siglo pasado, pero, a partir de los años 80 , se ha reconocido su papel como un patógeno causante de enfermedades transmitidas por alimentos en humanos (4). La bacteria está distribuida ampliamente en la naturaleza; además, es posible aislarla de animales y humanos sanos, portadores (4).

Listeria monocytogenes tiene una gran habilidad para sobrevivir y crecer bajo condiciones adversas,

\section{Correspondencia:}

Ángela Bibiana Muñoz, Calle $160 \mathrm{~N}^{\circ} 60-07$, torre 10, apartamento 1101, Bogotá, D.C., Colombia

Teléfono: (571) 220 7700, extensión 1421

angelamunoz7@hotmail.com

Recibido: 02/05/12; aceptado:18/02/13 incluso en refrigeración, razón por la cual puede transmitirse por consumo de una amplia variedad de alimentos, particularmente en aquellos listos para el consumo (5). Este agente patógeno tiene cada vez mayor importancia en salud pública a nivel mundial y se conocen datos epidemiológicos de algunos países desarrollados, como Estados Unidos, en donde se considera como el factor etiológico responsable de, aproximadamente, 2.500 casos y 500 muertes al año (5).

La listeriosis, enfermedad causada por L. monocytogenes, es una de las principales zoonosis emergentes de transmisión alimentaria (6); se considera como la infección de origen alimentario con mayor tasa de letalidad (20 a $30 \%$ de los casos), que evoluciona de casos esporádicos que pueden incrementarse a pequeños brotes e, incluso, consolidarse en verdaderas epidemias (2). Se estima que la excreción en materia fecal de $L$. monocytogenes es menor del $1 \%$; en contactos y pacientes asintomáticos es del $26 \%$ y en trabajadores de plantas de sacrificio es de 4,8\% (4), razón por la cual su aislamiento en materia fecal es compleja pero muy útil para la detección de portadores de la bacteria.

Listeria monocytogenes, además de causar graves infecciones en los seres humanos, también se encuentra causando enfermedad invasiva en muchas especies de diferentes de animales, incluidos los rumiantes de granja, como vacas, ovejas y cabras (7). Varios productos alimenticios derivados de estos animales, han sido frecuentemente implicados como fuentes de casos y brotes de listeriosis en humanos (8), ya que el microorganismo presente en las materias primas se puede inactivar eficazmente por las combinaciones de tiempo y temperatura típicas para la producción de alimentos más procesados (por ejemplo, leche pasteurizada), pero es difícil de manejar en productos alimenticios listos para el consumo, los cuales sufren contaminación cruzada con elementos contaminados del entorno de la planta de procesamiento (9) o con las manos de los manipuladores durante el proceso de producción, lo cual se constituye como la primera causa de 
generación de enfermedades transmitidas por alimentos (10). Los trabajadores relacionados con la producción de alimentos derivados de origen bovino se encuentran en permanente contacto con las materias primas y con los productos terminados, lo que los convierte en el personal de mayor riesgo de portar este microorganismo y de transmitirlo a los alimentos listos para consumo.

Por esta razón, el objetivo de este estudio fue determinar la prevalencia de $L$. monocytogenes a partir de muestras de materia fecal y frotis de manos de manipuladores de alimentos relacionados con la producción de lácteos y cárnicos en diez departamentos de Colombia y buscar asociación entre la presencia del microorganismo y los posibles factores de riesgo.

\section{Material y métodos}

Se planteó un estudio descriptivo de corte transversal para determinar la prevalencia de $L$. monocytogenes en manipuladores de alimentos mediante la recolección de muestras de materia fecal y frotis de manos. La población blanco del estudio estuvo conformada por manipuladores de alimentos de establecimientos dedicados a la producción de derivados lácteos y cárnicos de origen bovino, ubicados en 10 departamentos de Colombia. Para la selección de la muestra se tuvo en cuenta el volumen de producción de estos alimentos por cada departamento durante el año 2009 y el número de establecimientos autorizados para operar en cada región, garantizando de esta forma que se selccionara una muestra balanceada mediante la asignación proporcional. Los departamentos seleccionados para la toma de muestra fueron: Antioquia, Atlántico, Boyacá, Caquetá, Córdoba, Cundinamarca, Meta, Nariño, Santander y Valle del Cauca.
Para la selección de los establecimientos en cada departamento, se tuvieron en cuenta aquellos que estaban dedicados a actividades productivas de derivados lácteos y cárnicos, y que, además, se encontraban registrados ante el Instituto Nacional de Medicamentos y Alimentos (INVIMA) que es la autoridad sanitaria nacional encargada de la vigilancia de la producción de alimentos y medicamentos en el país. Un censo suministrado por esta entidad sirvió como insumo para el cálculo del tamaño de la muestra, para lo cual se tuvo en cuenta el número de establecimientos por departamento y por actividad productiva, con un nivel de confianza del $95 \%$ y una prevalencia esperada del $0,8 \%$. Esta prevalencia fue tomada de la única publicación encontrada durante la revisión, que reporta $L$. monocytogenes en manipuladores de alimentos sanos (11). El cálculo se hizo con el software Epi-Info 7 y el número de establecimientos visitados por cada actividad productiva y por cada departamento, se muestra en el cuadro 1.

Los establecimientos que ingresaron al estudio se seleccionaron usando un sistema de números aleatorios y, además, se tuvo en cuenta que los representantes legales de cada establecimiento expresaran mediante un acta de compromiso su consentimiento para participar voluntariamente en el estudio; si alguno se negaba, se continuaba con el siguiente hasta completar el número de muestras por cada departamento y por cada actividad productiva.

Para la selección de los manipuladores de alimentos de cada empresa, se aplicó un muestreo por cuota, estableciendo tres subgrupos: 1) empresas pequeñas (hasta 5 manipuladores), 2) empresas medianas (hasta 20 manipuladores), y 3) empresas grandes (más de 20 manipuladores), en donde cada

Cuadro 1. Número de establecimientos por departamento y tipo de producto

\begin{tabular}{lccc}
\hline Departamento & $\begin{array}{c}\text { Establecimientos de } \\
\text { leche } \mathbf{y} \text { derivados }\end{array}$ & $\begin{array}{c}\text { Establecimientos de } \\
\text { derivados cárnicos }\end{array}$ & Total \\
\hline Antioquia & 9 & 22 & 31 \\
Caquetá & 12 & 2 & 12 \\
Córdoba & 7 & 2 & 9 \\
Cundinamarca & 23 & 3 & 26 \\
Nariño & 32 & 8 & 40 \\
Atlántico & 6 & 7 & 13 \\
Boyacá & 11 & 4 & 15 \\
Meta & 13 & 3 & 16 \\
Santander & 11 & 12 & 23 \\
Valle del Cauca & 13 & 7 & 20 \\
Total & 137 & 70 & 207 \\
\hline
\end{tabular}

Fuente: PLE-INS (base de datos en CD-ROM actualizada cada mes durante 2010 y 2011) 
subgrupo aportó el $100 \%$, el $50 \%$ y el $20 \%$ de sus trabajadores, respectivamente; en cada grupo se aplicó un muestreo por conveniencia, teniendo en cuenta los trabajadores que se encontraban laborando en el momento de la visita a la empresa y que desarrollaban actividades específicas de manipulación directa de alimentos.

De cada manipulador de alimentos seleccionado, se recolectó una muestra de material fecal y un frotis de manos; además, a cada uno se le aplicó un cuestionario para identificar algunos factores de riesgo relacionados con la presencia de L. monocytogenes. El cuestionario constaba de cuatro partes: factores socio-demográficos, signos y síntomas relacionados con el evento, hábitos higiénicos, y conocimiento y aplicación de medidas de desinfección en áreas de trabajo. La mayoría de las preguntas fueron cerradas, pero algunas se dejaron abiertas para permitir que el manipulador pudiera ampliar sus respuestas; además, algunas opciones de respuesta que indagaban sobre hábitos higiénicos y medias de desinfección se corroboraron mediante la observación directa y entrevista del encuestador. Todos los manipuladores de alimentos respondieron el cuestionario de manera individual y sin la presencia de los jefes o representantes legales de la empresa, y estos últimos no conocieron las respuestas suministradas por sus trabajadores.

Un total de 1.322 muestras de materia fecal e igual número de muestras de frotis de manos, fueron recolectadas y enviadas en medio de transporte y condiciones de refrigeración al laboratorio del Grupo de Microbiología del Instituto Nacional de Salud, en donde se llevaron a cabo las análisis microbiológicos para la detección de L. monocytogenes, para lo cual se hicieron un enriquecimiento selectivo en caldo Fraser y un cultivo selectivo en agar Oxford y agar Palcam (12).

La identificación de las colonias sospechosas se hizo por coloración de Gram y pruebas bioquímicas, como prueba de CAMP (Christie, Atkins and MunchPetersen), catalasa, fermentación de azúcares (xilosa, ramnosa y manitol), esculina y motilidad a 25 y $37^{\circ} \mathrm{C}$, usando como control positivo la cepa $L$. monocytogenesATCC 19118 y, como control negativo, la cepa Staphylococcus aureus ATCC 25923.

En los aislamientos que fueron identificados como L. monocytogenes, se hicieron pruebas de sensibilidad antimicrobiana frente a penicilina, ampicilina y trimetoprim-sulfametoxazol, utilizando la técnica de microdilución en placa y siguiendo las recomendaciones del Clinical and Laboratory Standards Institute (CLSI) 2011 (13).

Para el análisis estadístico de los datos, se utilizó el sistema SPSS ${ }^{\mathrm{TM}}$, Statitics, versión 20, por medio del cual se determinaron medidas de frecuencia y de tendencia central para las variables sociodemográficas y de resultado positivo para el microorganismo. Además, se uso el test de $\chi^{2}$ para establecer relaciones entre variables consideradas como factores de riesgo y los resultados de las pruebas de laboratorio, y se consideraron significativas con valores de $p<0,05$. Para determinar si las condiciones sobre las que se indagaba en el cuestionario podrían asociarse como posibles factores de riesgo de ser portador de L. monocytogenes en nuestra población, primero se hizo un análisis bivariado y, posteriormente, uno multivariado por regresión logística para aquellas variables que resultaron significativas $(p<0,05$; $\mathrm{OR}>1, I_{95 \%}$ ) en el análisis bivariado inicial.

Se definieron como variables relevantes para la búsqueda de posibles factores de riesgo: no usar uniforme, no tener las manos limpias con uñas cortas y sin esmalte, no haber recibido capacitación en prácticas higiénicas, consumir alimentos en el área de trabajo, no lavarse las manos con frecuencia, no conocer el concepto de contaminación cruzada (por ende, no aplicarlo), no disponer en la empresa de lavamanos con jabón y toallas de papel, no practicar procedimientos adecuados de limpieza y desinfección, y no conocer las concentraciones y formas correctas de usar los desinfectantes.

Luego del análisis multivariado, las variables que mostraron una asociación estadísticamente significativa con el estado de portador de $L$. monocytogenes, fueronanalizadasestratificándolas por los diferentes datos demográficos de los portadores, tales como edad, sexo, nivel de educación y antigüedad laboral.

\section{Resultados}

Se recolectaron 1.322 muestras de cada uno de los especímenes (frotis de manos y materia fecal) de los 1.322 manipuladores de alimentos incluidos; se excluyeron del estudio aquellos manipuladores que por alguna razón no permitieran la recolección de los dos tipos de muestra.

Se encontraron 138 (10,4\%) manipuladores de alimentos positivos para L. monocytogenes (cuadro 2), de los cuales, $92(66,7 \%)$ fueron positivos únicamente en el frotis de manos, 40 (29\%) fueron 
positivos solo en la muestra de materia fecal y 6 $(4,3 \%)$ lo fueron en ambas muestras.

Para el análisis de las características de los manipuladores y de factores de riesgo, de aquí en adelante se denominan "portadores" a los manipuladores de alimentos que resultaron positivos para cualquiera de las dos muestras o para ambas.

El análisis de sensibilidad antibiótica de los aislamientos de L. monocytogenes recuperados de los manipuladores de alimentos, evidenció $100 \%$ de sensibilidad frente a los antibióticos probados.

Se encontró L. monocytogenes en todos los departa-mentos y el que presentó mayor número de manipuladores de alimentos con resultados positivos fue Nariño ( $31,2 \%)$, mientras que el que presentó el menor número fue Córdoba $(0,8 \%)$ (cuadro 2, figura 1).

Los establecimientos participantes con resultados positivos, fueron clasificados según su ubicación, y se encontró que el $80,5 \%$ de ellos estaban en la zona urbana y el $19,5 \%$ en la zona rural; en cuanto a las características demográficas de los portadores de L. monocytogenes, se encontró que el $79 \%$ eran hombres, el promedio de edad fue de 32 años (incluyendo tres menores de edad y un adulto mayor de 65 años) y el $55 \%$ tenía formación en educación secundaria. En cuanto a los años de experiencia laboral, se evidenció que el rango con mayor frecuencia fue de 1 a 5 años de antigüedad laborando en establecimientos de este tipo; el $56 \%$ de los portadores de L. monocytogenes pertenecía a empresas productoras de derivados lácteos y el 44 \% a empresas productoras de derivados cárnicos.
En el análisis bivariado se encontró asociación estadísticamente significativa $(p<0,05 ; \quad O R>1$, $\mathrm{IC}_{95 \%}$ ) entre casi todas las variables definidas como posible factor de riesgo y el estado de portador de L. monocytogenes, a excepción de "No usar uniforme y consumir alimentos en el área de trabajo" (cuadro 3). Por esta razón, estas dos variables se excluyeron del análisis multivariado (cuadro 4), en el cual se encontró que solo las variables: "No conocer el concepto de contaminación cruzada" $\left(\mathrm{OR}\left(\mathrm{IC}_{95 \%}\right)=1,518 ; \mathrm{p}=0,004\right)$ y "No practicar procedimientos de limpieza y desinfección adecuados" (OR $($ IC95\%)=1,292; p=0,005) mostraron asociación estadísticamente significativa con el estado de portador de L. monocytogenes y pueden considerarse como posible factor de riesgo para la población estudiada.

Estas dos variables se analizaron nuevamente, pero esta vez estratificándolas por los datos demográficos de los portadores. El único dato demográfico que mostró asociación estadísticamente significativa fue el nivel de educación, con asociación entre los portadores que tenían nivel de educación primaria o secundaria y los que no conocían el concepto de contaminación cruzada $(p=0,004)$, así como con los que no aplicaban procedimientos adecuados de limpieza $(p=0,001)$.

\section{Discusión}

Este estudio permitió obtener una prevalencia de $L$. monocytogenes en manipuladores de alimentos de empresas dedicadas a la producción de derivados lácteos y cárnicos. Asimismo, permitió asociar prácticas y conductas de los manipuladores de alimentos como factores de riesgo del estado de

Cuadro 2. Prevalencia de Listeria monocytogenes en los departamentos seleccionados

\begin{tabular}{lccc}
\hline Departamento & $\begin{array}{c}\text { Muestras de frotis de } \\
\text { manos y materia fecal }\end{array}$ & $\begin{array}{c}\text { Positivos para } \\
\text { Lonocytogenes }(\mathbf{n})\end{array}$ & $\begin{array}{c}\text { Prevalencia de } \\
\text { L. monocytogenes (\%) }\end{array}$ \\
\hline Antioquia & 190 & 20 & 10,5 \\
Caquetá & 116 & 9 & 7,7 \\
Córdoba & 116 & 1 & 0,8 \\
Cundinamarca & 144 & 13 & 9 \\
Nariño & 157 & 49 & 31,2 \\
Atlántico & 101 & 5 & 5 \\
Boyacá & 110 & 10 & 9 \\
Meta & 74 & 6 & 1,3 \\
Santander & 136 & 24 & 4,4 \\
Valle del Cauca & 178 & 138 & 13,4 \\
Total & 1.322 & & 10,4 \\
\hline
\end{tabular}

Fuente: PLE-INS (base de datos en CD-ROM actualizada cada mes durante 2010 y 2011) 


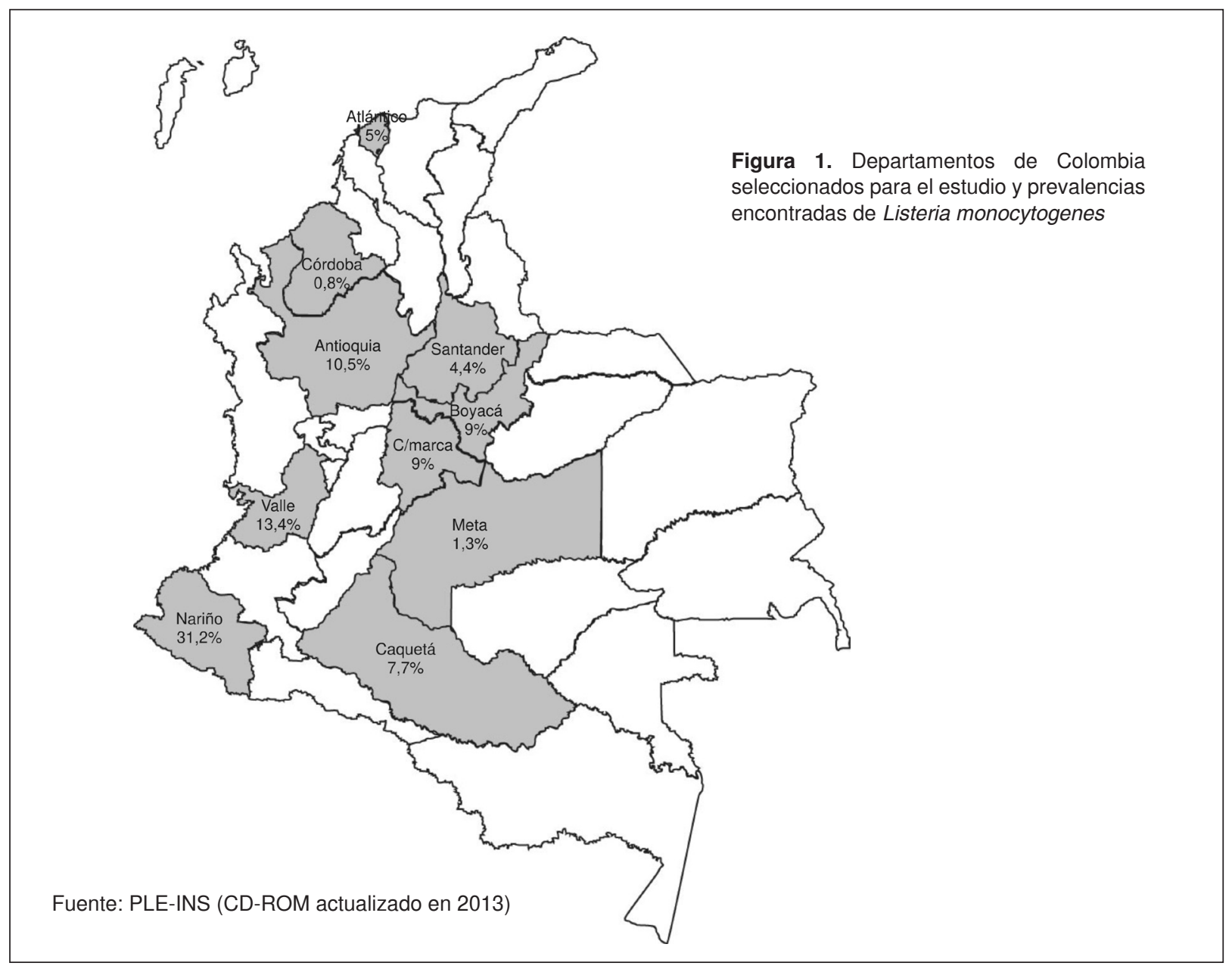

Cuadro 3. Análisis bivariado de variables incluidas como posibles factores de riesgo para portadores de Listeria monocytogenes

\begin{tabular}{|c|c|c|c|c|c|}
\hline \multirow[b]{2}{*}{ Variable } & \multirow[b]{2}{*}{$\chi^{2}$} & \multirow[b]{2}{*}{$\mathbf{p}$} & \multirow[b]{2}{*}{ OR } & \multicolumn{2}{|c|}{ IC $95 \%$} \\
\hline & & & & Inferior & Superior \\
\hline No usar uniforme adecuado & 2,8 & 0,09 & 1,8 & 1,6 & 5,0 \\
\hline No tener las manos limpias, uñas cortas y sin esmalte & 22,3 & 0,00 & 3,1 & 3,0 & 8,6 \\
\hline Sin capacitación en prácticas higiénicas & 39,7 & 0,00 & 3,7 & 2,1 & 6,4 \\
\hline Consume alimentos en área de trabajo & 3,4 & 0,06 & 1,7 & 2,0 & 4,3 \\
\hline No se lava las manos cada vez que sea necesario & 13,7 & 0,00 & 4,4 & 1,8 & 10,6 \\
\hline No sabe qué es contaminación cruzada & 34,6 & 0,00 & 2,9 & 1,3 & 3,3 \\
\hline No hay lavamanos con jabón y toallas de papel & 23,4 & 0,00 & 3,6 & 2,4 & 5,6 \\
\hline No practica procedimientos de limpieza y desinfección & 45,6 & 0,00 & 5,1 & 1,9 & 5,2 \\
\hline No conoce concentraciones y formas de usar los desinfectantes & 14,6 & 0,00 & 2,8 & 0,8 & 3,6 \\
\hline
\end{tabular}

Fuente: Resultado 1 Listeria (CD-ROM actualizado el 15 de abril de 2012)

portador. Por lo tanto, se convierte en una herramienta útil para la caracterización epidemiológica de $L$. monocytogenes, apoya el fortalecimiento de la vigilancia en salud pública de las enfermedades transmitidas por alimentos y encamina las estrategias para la reducción de la contaminación de alimentos con esta bacteria. Las estrategias deben implementarse prontamente teniendo en cuenta que entre 1995 y 2009 se identificaron en el mundo 11 brotes de listeriosis asociados al consumo de quesos, con 545 personas afectadas, con tasas de mortalidad estimadas entre el 14 y $30 \%$ y, de hospitalización, hasta del $100 \%$; y, aunque en Colombia no existen datos similares, sí se supo, en el reporte al Sistema Nacional de Vigilancia en Salud Pública (SIVIGILA) de 2009, de un caso con manifestaciones de meningitis en un niño de nueve años, debido al consumo de queso fresco contaminado (14). 
Cuadro 4. Análisis multivariado de variables incluidas como posibles factores de riesgo para portadores de L. monocytogenes

\begin{tabular}{|c|c|c|c|c|c|}
\hline \multirow[t]{2}{*}{ Variable } & \multirow[t]{2}{*}{ Prueba de Wald } & \multirow[t]{2}{*}{$\mathbf{p}$} & \multirow[t]{2}{*}{ OR } & \multicolumn{2}{|c|}{ IC 95\% para OR } \\
\hline & & & & Inferior & Superior \\
\hline No tener las manos limpias, uñas cortas y sin esmalte & 1,969 & 0,161 & 0,643 & 0,347 & 1,191 \\
\hline Sin capacitación en prácticas higiénicas & 2,552 & 0,110 & 0,621 & 0,346 & 1,114 \\
\hline No se lava las manos cada vez que sea necesario & 0,652 & 0,420 & 0,657 & 0,237 & 1,823 \\
\hline No sabe qué es contaminación cruzada & 8,318 & 0,004 & 1,518 & 1,232 & 1,910 \\
\hline No hay lavamanos con jabón y toallas de papel & 0,107 & 0,744 & 1,144 & 0,511 & 1,362 \\
\hline No práctica procedimientos de limpieza y desinfección & 7,787 & 0,005 & 1,292 & 1,023 & 1,993 \\
\hline No conoce concentraciones y formas de usar los desinfectantes & 1,126 & 0,289 & 1,587 & 1,176 & 3,723 \\
\hline
\end{tabular}

Fuente: Resultado1 Listeria (CD-ROM actualizado el 15 de abril de 2012)

Los datos reportados en este estudio son, entonces, un primer acercamiento para avanzar en el conocimiento del papel de los manipuladores de alimentos en la contaminación de productos lácteos y cárnicos con L. monocytogenes, teniendo en cuenta que a nivel nacional no existen reportes similares y a nivel mundial son pocos las publicaciones al respecto. Los antecedentes que existen corresponden a una investigación en manipuladores de alimentos que reportó una prevalencia de 0,8\% (11), una cifra baja comparada con los hallazgos obtenidos en el presente estudio $(10,4 \%)$, lo cual plantea una alarma en cuanto a la situación sanitaria y de salud de los manipuladores de alimentos en Colombia. Además, esta prevalencia de $10,4 \%$ es similar a las de $L$. monocytogenes en alimentos lácteos y cárnicos en Colombia (3,15,16; Vera HI, Ferro CJ, Triana LM. Prevalencia de Listeria monocytogenes en derivados cárnicos cocidos para consumo directo analizados en el Laboratorio de Salud Pública Distrito de Bogotá 1 de septiembre 2001 31 de agosto 2004. I Congreso Nacional de Salud Pública. Bogotá: Pontificia Universidad Javeriana; 23 al 25 de octubre de 2006), que oscilan entre el 10 $\%$ y el $16 \%$, lo que pone de manifiesto la necesidad de incluir la búsqueda de $L$. monocytogenes en las pruebas de coprocultivo requeridas para todo manipulador de alimentos en Colombia antes de su ingreso a los establecimientos de la industria alimentaria. Además, muestra que es necesario encaminar estrategias para dilucidar los posibles mecanismos involucrados en la contaminación de los alimentos y los manipuladores de alimentos con L. monocytogenes en Colombia.

En otra investigación sobre portadores de $L$. monocytogenes en personas sanas que no tenían contacto con materias primas de origen animal ni estaban relacionadas con la producción de alimentos (17), se reportó una prevalencia de $3 \%$, lo cual puede reflejar que el 10,4 \% encontrado aquí puede deberse a la presencia de una cadena de contagio entre las materias primas, el alimento terminado y los manipuladores de alimentos.

Los hallazgos de este estudio dejan ver la problemática en las cadenas productivas de lácteos y cárnicos. Sin embargo, es importante llamar la atención sobre la presencia de este microorganismo en todo tipo de alimentos, ya que cada vez más se reporta su presencia en otros grupos alimenticios debido a su tolerancia frente a diversas condiciones (18); incluso, L. monocytogenes fue reportado como agente causal de un brote que se presentó por la ingestión de melón, una fruta que usualmente no era considerada como un alimento de riesgo para la presencia de este tipo de microorganismos (19).

Los aislamientos obtenidos en este estudio mostraron ser $100 \%$ sensibles a los antibióticos de elección terapéutica (penicilina, ampicilina y trimetoprim-sulfametoxasol), muy similar a lo reportado en Brasil por Falavina, et al. (20), quienes analizaron 68 cepas aisladas de pacientes con listeriosis, todas las cuales mostraron ser sensibles a los antibióticos $\beta$-lactámicos y solo dos (3 \%) fueron resistentes a trimetoprim-sulfametoxasol. Aunque en varios estudios se evidencie, en general, baja resistencia a los antimicrobianos, es importante continuar el seguimiento de las cepas de L. monocytogenes frente a los antibióticos, teniendo en cuenta que frecuentemente se ha reportado su capacidad para adquirir genes de resistencia a partir de otras especies bacterianas (21) y que en la industria agroalimentaria se practica el uso de antimicrobianos como promotores de crecimiento en la producción de animales (22).

Entre las respuestas del cuestionario aplicado a los manipuladores de alimentos, se identificaron dos variables que pueden considerarse como posibles factores de riesgo para el estado de portador de 
L. monocytogenes en esta población: "No conocer el concepto de contaminación cruzada" y "No practicar procedimientos de limpieza y desinfección adecuados". Asimismo, estas dos variables mostraron una asociación estadísticamente significativa con el nivel de educación de los manipuladores, es decir, aquellos que no tenían formación técnica o universitariaeranquienesmayormentemanifestaban no conocer los conceptos ni la forma adecuada de desinfección, dejando, entonces, expuesto uno de los principales problemas en la industria alimentaria y que favorece la contaminación de los trabajadores y de los alimentos: deficiencias en la capacitación y seguimiento a los manipuladores de alimentos, por parte de las empresas.

La manipulación de los alimentos en las etapas productivas de los mismos es una parte crítica y, aunque no en todos los casos es necesario que los trabajadores encargados de esta tarea cuenten con estudios técnicos o universitarios, sí es vital para la producción que todo el personal se encuentre debidamente capacitado. El Decreto 3075 de 1997 del Ministerio de Salud de Colombia, reglamenta la obligatoriedad de que todas las personas que desempeñen actividades de manipulación de alimentos deben tener formación en materia de educación sanitaria, especialmente en cuanto a prácticas higiénicas en la manipulación de alimentos; asimismo, manifiesta que todas las empresas productoras de alimentos deben tener un plan de capacitación continuo y permanente para el personal manipulador desde el momento de su contratación, que después debe ser reforzado mediante charlas, cursos $u$ otros medios efectivos de actualización (23).

A partir de los resultados obtenidos en este estudio, se puede inferir que existen deficiencias en los programas de capacitación continua de dichos manipuladores por parte de las empresas o que no se cuenta con un programa de verificación de la apropiación del conocimiento; por esta razón, se presentan casos de manipuladores que no conocen el concepto de contaminación cruzada y se asume que, por ende, no lo aplican y tampoco practican procedimientos adecuados de limpieza y desinfección, lo cual se ha convertido en un riesgo para la inocuidad de los alimentos, la salud del consumidor y de los mismos manipuladores y de sus familias, ya que a través de ellos se puede diseminar $L$. monocytogenes a los demás miembros del hogar.

Además de evidenciar deficiencias en los procesos de capacitación a los manipuladores, este estudio resalta la importancia de que las empresas realicen procesos de actualización en sus medidas de desinfección de áreas y, también, de que promuevan en sus trabajadores la práctica adecuada de estas medidas, ya que se ha demostrado que $L$. monocytogenes es un microorganismo capaz de adherirse a las superficies que entran en contacto con los alimentos y formar biopelículas, las cuales dificultan su eliminación efectiva en los procesos de limpieza y desinfección (24).

Los alimentos más propensos a la contaminación con L. monocytogenes son los lácteos y los cárnicos, y el incorrecto manejo de las materias primas y la contaminación posterior de los alimentos, son las explicaciones más probables de la presencia de patógenos en los alimentos terminados y de la generación de casos y brotes de enfermedades transmitidas por alimentos (18). Por lo tanto, los hallazgos de este estudio brindan información importante, tanto por la demostración de portadores de este patógeno en manipuladores de alimentos, como por el aporte del análisis de factores de riesgo que, sumados, brindan un acercamiento a nuevos aspectos que todas las autoridades involucradas en la vigilancia de las cadenas productivas agroalimentarias del país deben tener en cuenta. Sin embargo, aún queda mucho por entender en lo que respecta a los aspectos epidemiológicos de L. monocytogenes, ya que es un problema sanitario en evolución que involucra muchas partes.

\section{Agradecimientos}

A todos los manipuladores de alimentos y a los representantes legales de las empresas seleccionadas por participar voluntariamente en el estudio y permitir la toma de muestra.

A la Subdirección de Alimentos y Bebidas Alcohólicas del INVIMA por su colaboración en el suministro de los censos de establecimientos.

A los Grupos de Trabajo Territorial INVIMA de: Costa Caribe 1, Costa Caribe 2, Centro Oriente 1, Centro Oriente 2, Centro Oriente 3, Occidente 1, Occidente 2 y Orinoquia, por su valiosa colaboración en el acompañamiento a las visitas a las empresas seleccionadas.

\section{Conflicto de intereses}

Los autores declaramos que, durante la realización del trabajo, no existió conflicto de intereses de ningún tipo. 


\section{Financiación}

Este trabajo fue financiado por el Instituto Nacional de Salud y por dineros del CONPES 3376 de política sanitaria y de inocuidad para las cadenas de la carne bovina y de la leche.

\section{Referencias}

1. Instituto Nacional de Salud. Subdirección de vigilancia y control en salud pública. Protocolo de vigilancia y control de enfermedades transmitidas por alimentos. Versión 00. Agosto de 2010. Fecha de consulta: 1 de septiembre de 2012. Disponible en: http://www.ins.gov.co/lineas-de-accion/ Subdireccion-Vigilancia/sivigila/Protocolos\%20SIVIGILA/ PRO-R02.003.0000-001\%20ETA.pdf

2. FAO/OMS. World Health Organization/Food and Agriculture Organization of the United Nation. Risk assessment of Listeria monocytogenes in ready-to-eat foods. Technical report. Microbiological risk assessment series 5. Rome, Italy. 2004. Fecha de consulta: 1 de septiembre de 2012. Disponible en: ftp://ftp.fao.org/docrep/fao/010/y5394e/y5394e.pdf

3. Campo A, Espinosa J, Vargas M, Álvarez C, Rodríguez $\mathbf{S}$, Chávez J, et al. Prevalencia de agentes patógenos asociados a grupos de alimentos de alto riesgo en salud pública, Colombia, 2007. Inf Quinc Epidemiol Nac. 2008;13:351-66

4. Muñoz Al, Díaz G. Listeriosis. Bogotá D.C.: INVIMA; 1998.

5. Medrano MV, Restrepo S, Vanegas MC. Tipificación molecular de Listeria monocytogenes aisladas de muestras clínicas y alimentos. Biomédica. 2006;26:442-50.

6. Hof H. History and epidemiology of listeriosis. FEMS. 2003;35:199-202. 10.1016/S0928-8244(02)00471-6

7. Nightingale KK, Schukken $\mathrm{YH}$, Nightingale CR, Fortes ED, Ho AJ, Her YT, et al. Ecology and transmission of Listeria monocytogenes infecting ruminants and in the farm environment. Appl Environ Microbiol. 2004;70:4458-67. http://dx.doi.org/10.1128/AEM.70.8.4458-4467.2004

8. Mead PS, Slutsker L, Dietz V, McCraig LF, Bresee JS, Shapiro C, et al. Food-related illness and death in the United States. Emerg Infect Dis. 1999;5:607-25.

9. Gravani R. Incidence and control of Listeria in foodprocessing facilities. En: Ryser ET, Marth EH, editors. Listeria listeriosis and food safety. 2nd edition. New York: Taylor \& Francis; 1999. p. 657-709.

10. Sánchez FJ, Mata V, Espinoza A, Villareal L. Incidencia de especies de Listeria en una planta productora de alimentos congelados. Ciencia UNAL. 2006;IX:51-6.

11. Müeller HE. Listeria isolations from feces of patients with diarrhea and from healthy food handlers. Infection. 1990;18:97-9. http://dx.doi.org/10.1007/BF01641423

12. WHO Global Sarm Surv. Manual de procedimientos. Aislamiento, identificación y caracterización de Listeria monocytogenes. 2008. Fecha de consulta: 1 de septiembre de 2012. Disponible en: http://publicaciones.ops.org. ar/publicaciones/cursos_virtuales/ETAsM3/bibliografia/ Manual\%20de\%20Listeria\%20monocytogenes\%202008. pdf
13. Clinical and Laboratory Standards Institute. Methods for antimicrobial dilutions and disk susceptibility testing of infrequently isolated or fastidious bacteria. Approved Guideline M45-A. Wayne: CLSI; 2006.

14. Instituto Nacional de Salud. Subdirección de Investigación. Evaluación de riesgos de Listeria monocytogenes en queso fresco en Colombia. 2011. Fecha de consulta: 12 de febrero de 2013. Disponible en: http://www.ins.gov.co/ lineas-de-accion/investigacion/ueria/Publicaciones/ ER\%20 LISTERIA\%20EN\%20LPC.pdf

15. Albarracin FY, Sarmiento P, Carrascal AK, Mercado M. Estimación de la proporción de Listeria monocytogenes y Salmonella spp. en quesos frescos (queso de hoja, cuajada) y queso doble crema producidos y comercializados en el municipio de Pamplona, Norte de Santander. BISTUA. 2006;4:30-42.

16. Muñoz Al, Vargas M, Otero L, Díaz G, Guzmán V. Presencia de Listeria monocytogenes en alimentos listos para el consumo, procedentes de plazas de mercado y delicatessen de supermercados de cadena, Bogotá, D.C, 2002-2008. Biomédica. 2011;31:428-39.

17. Grif K, Patscheider G, Dierich MP, Allerberger F. Incidence of fecal carriage of Listeria monocytogenes in three healthy volunteers: A one-year prospective stool survey. Eur J Clin Microbiol Infect Dis. 2003;22:16-20. http:// dx.doi.org/10.1007/s10096-002-0835-9

18. Rossi ML, Paiva A, Tornese M, Chianelli S, Troncoso A. Brotes de infección por Listeria monocytogenes: una revisión de las vías que llevan a su aparición. Rev Chil Infectol. 2008;25:328-35. http://dx.doi.org/10.4067/S071610182008000500002

19. Centers for Disease Control and Prevention. Multistate outbreak of listeriosis associated with Jensen Farms cantaloupe -United States, August-September 2011. MMWR Morb Mortal Wkly Rep. 2011;60:1357-8.

20. Falavina CM, Barbosa AV, Alves L, Vallim DC, Hofer E. Antimicrobial susceptibilities of Listeria monocytogenes human strains isolated from 1970 to 2008 in Brazil. Rev Soc Bras Med Trop. 2011;44:173-6. http://dx.doi.org/10.1590/ S0037-86822011005000019

21. Ruiz Z, Neuque MC, Poutou RA, Carrascal AK, Mattar S. Antimicrobial susceptibility of Listeria monocytogenes food isolates from different cities in Colombia. Foodborne Pathog Dis. 2011;8:913-9. http://dx.doi.org/10.1089/fpd.2010.0813

22. Ruiz Z, Poutou RA, Carrascal AK. Resistencia antimicrobiana y a desinfectantes de Listeria spp. NOVA. 2008;6:201-18.

23. Ministerio de Salud de Colombia. Decreto 3075 de 1997. Fecha de consulta: 1 de septiembre de 2012. Disponible en: http://www.legicol.com/lejuro40/Decreto_3075_de_1997. pdf

24. Aase B, Sundheim G, Langsrud S, Rorvik LM. Occurrence of and a possible mechanism for resistance to a quaternary ammonium compound in Listeria monocytogenes. Int $\mathrm{J}$ Food Microbiol. 2000;62:57-63. 